\title{
Thrombosis of the internal jugular vein
}

\author{
Lloyd Isaac Tannenbaum ${ }^{1} \cdot$ Michael David April ${ }^{1} \cdot$ Robert Edward Watts $^{1} \cdot$ \\ Nadia Mary Pearson ${ }^{1}$
}

Received: 4 August 2015/Accepted: 19 August 2015/Published online: 5 September 2015

(C) SIMI (outside the USA) 2015

\begin{abstract}
A 35-year-old woman presented to our Emergency Department with a gradual-onset occipital headache radiating into her neck, and worsening over the prior 6 days. She reported inability to move her neck due to pain and stiffness. She denied trauma, fevers, or other positive findings on review of systems. She further denied any history of prior similar headaches. Past medical history included a history of stage 1 renal cell carcinoma treated with right nephrectomy 5 years prior. She took no medications. Family history was unremarkable. Social history was significant for tobacco use.
\end{abstract}

On physical examination, her blood pressure was $145 / 88 \mathrm{mmHg}$, and all other vital signs were within normal limits. The patient did not tolerate any neck motion secondary to pain. There was tenderness over the para-cervical musculature bilaterally, without any bony tenderness. The neurologic examination was normal to include negative Kernig's and Brudzinski's signs.

Based on this headache presentation with neck involvement in the absence of a history of trauma, osseous injury and intracranial bleeding were both thought to be unlikely. In light of the patient's new gradual-onset headache with a history of malignancy and tobacco use and now

The view(s) expressed herein are those of the author(s) and do not reflect the official policy or position of Brooke Army Medical Center, the U.S. Army Medical Department, the U.S. Army Office of the Surgeon General, the Department of the Army and Department of Defense or the U.S. Government.

Michael David April

Michael.D.April@post.harvard.edu

1 Departments of Emergency Medicine and Radiology, San Antonio Uniformed Services Health Education Consortium, 3551 Roger Brooke Dr, JBSA, Fort Sam Houston,

San Antonio, TX 78234-6200, USA inability to move her neck, our differential diagnosis at this time prioritized vascular thrombosis and neoplasm. Given an absence of trauma or neurologic findings, we reasoned arterial dissection to be less likely. Consequently, to optimize evaluation for neoplasm and venous thrombosis, we ordered computed tomography (CT) of the head and neck with intravenous contrast in the venous instead of arterial phase (Fig. 1). Alternatively, arterial pathology (dissection) would have been better evaluated using arterial phase contrast, if it had been higher on our differential.

The CT scan demonstrated an occlusive right internal jugular and dural venous thrombosis. The patient was anticoagulated with unfractionated heparin, and admitted. Serial CT imaging of the head and subsequent MRI of the brain revealed no interval changes of the venous thrombosis. Further work-up for malignancy or hypercoagulability was only notable for a positive Lupus anticoagulant test. She was discharged on rivaroxaban. Her symptoms improved, and she experienced no complications as of 6-month follow-up post-discharge.

There is substantial consensus regarding when to obtain emergent non-contrast CT imaging of the head in patients presenting with headache. The most dangerous disease entity of interest in such patients is generally intracranial bleeding, for which head CT scan without contrast has excellent sensitivity [1]. Similarly, there is widespread consensus regarding when to obtain emergent non-contrast cervical spine CT scan in patients presenting after blunt neck trauma. The most common deadly diagnoses in such patients are likely to be osseous spinal injuries, for which CT without contrast again is an optimal diagnostic tool [2].

Conversely, the indications for emergent head and neck imaging with contrast are less well established. This case suggests inability to move the neck as a potential indication 
Fig. 1 a Axial non-contrast computed tomography (CT) image of the head. Imaging demonstrates asymmetric hyper-density and expansion within the right transverse sinus (arrow), indicative of thrombus. b Sagittal CT image of the neck with venous phase contrast. Imaging demonstrates acute thrombus within the right IJ vein (arrow)
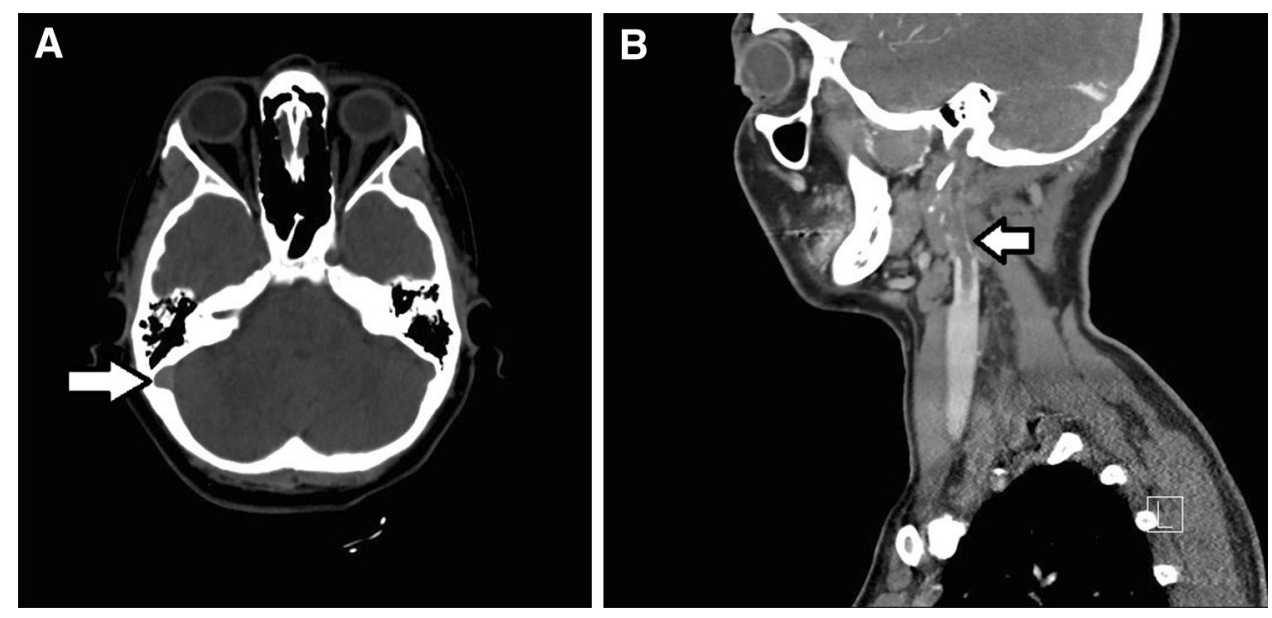

for neck imaging with intravenous contrast. As with all diagnostic considerations, the decision to order a contrastenhanced study is driven by differential diagnosis. Arterial pathology (dissection) is best evaluated using arterial phase contrast enhancement [3]. In the case of our patient, venous phase contrast enhancement was utilized to aid in identification of neoplasms and soft tissue infections amidst the complex array of tissue planes in the neck [3, 4]. Furthermore, we reasoned that the addition of venous phase contrast would aid in the identification of vascular thrombosis that was ultimately determined to be the cause of this patient's symptoms [4, 5]. We propose that physicians treating patients presenting with headache and inability to move their necks should broaden their differential diagnosis beyond acute bleeding and osseous injury and, therefore, consider neck CT scan with contrast enhancement to evaluate for additional disease entities including infection, neoplasm, and vascular dissection or thrombosis. The optimal choice of contrast phase will be driven by clinician assessment of whether arterial dissection (arterial phase) versus venous pathology, soft tissue infection, or neoplasm (venous phase) is considered more likely.

\section{Compliance with ethical standards}

Funding No fund received for this study.

Conflict of interest The authors declare that they have no conflict of interest.
Statement of human and animal rights All procedures performed in studies involving human participants were in accordance with the ethical standards of the institutional and/or national research committee and with the 1964 Helsinki declaration and its later amendments or comparable ethical standards. This article does not contain any studies with animals performed by any of the authors.

Informed consent Informed consent was obtained from all individual participants included in the study.

\section{References}

1. Douglas AC, Wippold FJ II, Broderick DF, Aiken AH, AminHanjani S, Brown DC, Corey AS, Germano IM, Hadley JA, Jagadeesan BD, Jurgens JS, Kennedy TA, Mechtler LL, Patel ND, Zipfel GJ (2013) Expert panel on neurologic imaging. ACR appropriateness criteria headache. American College of Radiology (ACR), Reston, p 23

2. Stiell I, Clement C, McKnight RD, Brison R, Schull M, Rowe B, Worthington J, Eisenhauer M, Greenberg G, MacPail I, Dreyer J, Lee J, Bandiera G, Reardon M, Holroyd B, Lesiuk H, Wells G (2003) The Canadian C-spine rule versus the NEXUS low-risk criteria in patients with trauma. N Engl J Med 349(26):2510-2518

3. Hudgins PA (1994) Contrast enhancement in head and neck imaging. Neuroimaging Clin N Am 4:101-115

4. Shook and Trigger (2014) Lemierre's syndrome. Western J Emerg Med 15(2):125-126

5. Efe SC, Poci N, Unkun T, Cap M, Acar RD, Gecmen C, Ozdemir N (2015) Nineteen-year-old female with idiopathic thrombosis of the internal jugular vein. Perfusion 30(4):341-344 